Revue d'histoire de l'Amérique française

REYUE D.HISTOIRE DE L'AMÉRIQUE FRANÇAISE

\title{
CHERWINSKI, W. J. C. et Gregory S. KEALEY, eds., Lectures in Canadian Labour and Working-Class History. Toronto, Committee on Canadian Labour History and New Hogtown Press, 1985. 198 p.
}

\section{Denyse Baillargeon}

Volume 40, numéro 3, hiver 1987

URI : https://id.erudit.org/iderudit/304472ar

DOI : https://doi.org/10.7202/304472ar

Aller au sommaire du numéro

Éditeur(s)

Institut d'histoire de l'Amérique française

ISSN

0035-2357 (imprimé)

1492-1383 (numérique)

Découvrir la revue

Citer ce compte rendu

Baillargeon, D. (1987). Compte rendu de [CHERWINSKI, W. J. C. et Gregory S. KEALEY, eds., Lectures in Canadian Labour and Working-Class History. Toronto, Committee on Canadian Labour History and New Hogtown Press, 1985. 198 p.] Revue d'histoire de l'Amérique française, 40(3), 434-436.

https://doi.org/10.7202/304472ar d'utilisation que vous pouvez consulter en ligne.

https://apropos.erudit.org/fr/usagers/politique-dutilisation/ 
CHERWINSKI, W. J. C. et Gregory S. KEALEY, eds., Lectures in Canadian Labour and Working-Class History. Toronto, Committee on Canadian Labour History and New Hogtown Press, 1985. 198 p.

En 1983 et 1984, le Programme d'études canadiennes du Secrétariat d'État finançait la tenue de quatre séries de conférences portant sur l'histoire du mouvement ouvrier et de la classe ouvrière canadienne. Le présent recueil réunit 
les textes de 14 des communications: huit proviennent de la première série de conférences qui s'est déroulée à l'Université Memorial de Terre-Neuve tandis que les six autres ont été choisies parmi celles présentées à l'Université Laval et à l'Université de Winnipeg.

Les séries de conférences, ainsi que le livre qui leur fait suite, avaient pour but de rendre la recherche dans ce domaine plus accessible aux travailleurs canadiens et au public en général. Les articles qui composent ce recueil s'intéressent donc pour la plupart à des questions relativement larges et couvrent souvent de longues périodes. Tout en faisant état des nouvelles problématiques en histoire du travail, plusieurs textes constituent une mini-synthèse du sujet abordé et s'avèrent donc fort utiles pour les néophytes et les étudiants. La répartition des textes dans le temps et l'espace privilégie le $20 \mathrm{e}$ siècle tout en témoignant du souci des éditeurs de faire connaître l'histoire régionale trop souvent négligée dans les ouvrages de synthèse.

En guise d'introduction, on retrouve deux survols portant sur l'histoire du mouvement ouvrier et de la classe ouvrière canadienne aux $19 \mathrm{e}$ et $20 \mathrm{e}$ siècles. Dans un historique centré sur les institutions, Eugène Forsey souligne d'abord les constantes qui se dégagent des luttes menées par les travailleurs pour ensuite dresser un bilan des organisations syndicales et politiques dont ils se sont dotés pour défendre leurs intérêts. Le texte de Kealey, qui lui fait pendant, se rattache au courant historiographique plus récent qui s'intéresse à l'ensemble de la classe ouvrière plutôt qu'à ses seules institutions. L'auteur examine la formation et l'évolution de la classe ouvrière canadienne à partir d'un découpage en cinq périodes correspondant à différentes phases du développement du capitalisme dont les crises constitueraient les dates charnières. S'inspirant largement des théories de Gordon, Edward et Reich, l'article vise à une meilleure intégration de l'histoire de la classe ouvrière et permet de mieux comprendre l'évolution des structures syndicales évoquées par Forsey.

Les deux articles qui traitent du 19e siècle nous le font voir à travers deux problématiques fort différentes. Michael Cross s'intéresse pour sa part au travail en tant que phénomène social définissant des pratiques individuelles (You are how you work) autant que les rapports de classes. Son article vise donc à cerner l'impact de la révolution industrielle sur les individus. De son côté, Bryan Palmer, tout comme Kealey, analyse le développement et la formation de la classe ouvrière au 19e siècle. Mais alors que Kealey se fonde principalement sur les changements dans les procès de travail, Palmer met en rapport le développement du capitalisme canadien et les signes d'une présence ouvrière (grèves et organisations syndicales); suivant son analyse, les années 18801900 représenteraient l'aboutissement d'un processus amorcé vers 1850 , l'accroissement spectaculaire des conflits et des adhésions syndicales marquant l'émergence d'une classe ouvrière cohérente et davantage solidaire.

La troisième partie de l'ouvrage, consacrée au 20e siècle, s'ouvre sur les articles de Joan Sangster et Joy Parr qui retracent l'évolution du travail salarié féminin en relation avec le travail domestique des femmes. Ce dernier devient un facteur essentiel dans l'analyse et la compréhension de l'expérience vécue par les femmes sur le marché du travail, qu'il s'agisse de la discrimination salariale, des ghettos d'emploi ou de leur faible taux de syndicalisation. Les deux synthèses témoignent des nouvelles problématiques introduites en his- 
toire des travailleuses par les recherches en histoire des femmes et intègrent les nouvelles interprétations qui en découlent. On peut toutefois déplorer que l'espace réservé à cette question soit occupé par deux textes qui se recoupent à maints égards.

A l'exception du texte de Robert Babcock, qui nuance son interprétation de la prise de contrôle par la Fédération américaine du travail sur le mouvement ouvrier canadien, le reste du recueil se compose d'articles consacrés aux travailleurs des différentes provinces. L'Ouest et les provinces maritimes font l'objet de cinq textes allors que le Québec et l'Ontario sont couverts par un seul article chacun. Cette répartition inhabituelle rend sans doute justice aux Maritimes, parent pauvre de l'histoire ouvrière canadienne, mais dessert le Québec encore mal connu au Canada anglais.

Ross McCormack signe les deux articles traitant des travailleurs de l'Ouest. Dans «Wobblies and Blanketstiffs», il retrace la montée et le déclin des «Industrial Workers of the World», en relation avec le développement économique de la région et la composition ethnique de la force de travail, cette dernière devenant un des facteurs expliquant sa disparition après la première guerre mondiale. Son deuxième texte, "The Western Working-Class Experience», reprend les mêmes éléments (développement économique accéléré et diversité ethnique) pour faire l'analyse des tendances radicales présentes dans l'ouest avant, mais aussi après la grève de Winnipeg. Pour l'auteur en effet, ce radicalisme a continué de s'exprimer par l'élection de gouvernements sociaux-démocrates, la tendance travailliste (labourism) étant ici interprétée comme radicale.

Les articles portant sur les travailleurs des Maritimes cherchent surtout à démontrer qu'il existe également une tradition militante et même radicale dans cette région du pays généralement perçue comme conservatrice et peu industrialisée. A l'aide de sources provenant des syndicats locaux, Ian McKay, par exemple, soutient que la philosophie conservatrice des dirigeants du Provincial Workmen's Association a souvent été contredite par le militantisme de la base. Cette interprétation n'est pas sans rappeler celle que Jacques Rouillard défendait à propos de la CTCC; reste à savoir jusqu'où on peut dissocier discours et pratique lorsqu'il s'agit d'une même organisation.

Enfin les textes de Léo Roback (auquel le livre est dédié) et de Bryan Palmer font la synthèse des luttes et des organisations syndicales au Québec et en Ontario depuis le début du 20e siècle. Celui de Roback, divisé en trois parties, insiste notamment sur la composition ethnique de la force de travail et souligne les particularités linguistiques et religieuses qui ont conduit à l'émergence d'organisations syndicales indépendantes du mouvement ouvrier canadien.

Ce recueil démontre bien la vitalité de ce champ de la recherche historique. L'intégration des nouvelles problématiques et des nouvelles interprétations en fait un outil fort utile pour l'enseignement de cette discipline malgré l'absence de références et d'une bibliographie. 